\title{
利用菊池衍射花样鉴定晶体结构的方法研究
}

$$
\text { 彭 帆 }{ }^{1,2} \text {, 曾 毅 }{ }^{1,2}
$$

(1. 中国科学院 上海硅酸盐研究所, 高性能陶瓷和超微结构国家重点实验室, 上海 200050; 2. 中国科学院大学 材料与光电研究中心, 北京 100049)

摘 要: 电子背散射衍射(Electron Backscatter Diffraction, EBSD)是研究材料显微结构的重要手段之一, 通过 EBSD 获取的菊池衍射花样是材料内部微观晶体结构的直观反映。本研究通过识别菊池花样中的对称轴, 结合晶体对称定 律, 提出了一种利用菊池花样进行晶体对称性分析和晶体结构鉴定的方法。通过该方法成功对三个未知样品的对称 性和晶体结构进行了判断。其中一个样品确定到所属晶系, 另两个样品锁定到部分点群, 通过确定晶系和点群排除 了部分不符合对称性的相鉴定结果。研究结果表明，利用菊池花样进行对称性分析是判断晶体结构的有效方法。同 现有方法相比, 菊池衍射花样法大大缩小了相鉴定的检索范围, 显著提高了相鉴定的准确性和可靠性, 是一种有 望用于新一代 EBSD 设备的标定技术。

关 键 词: EBSD; 菊池花样; 晶体结构鉴定; 相鉴定

中图分类号: TQ174 文献标志码: A

\section{Method of Crystal Structure Identification by Using Kikuchi Diffraction Patterns}

\author{
PENG Fan $^{1,2}$, ZENG Yi ${ }^{1,2}$
}

(1. The State Key Lab of High Performance Ceramics and Superfine Microstructure, Shanghai Institute of Ceramics, Chinese Academy of Sciences, Shanghai 200050, China; 2. Center of Materials Science and Optoelectronics Engineering, University of Chinese Academy of Sciences, Beijing 100049, China)

Abstract: Electron backscatter diffraction (EBSD) is an important microstructure analysis method in the field of inorganic materials research. The Kikuchi pattern obtained through EBSD is an intuitive reflection of the internal microscopic crystal structure of the material. In this study, a method of crystal symmetry analysis and crystal structure identification by using Kikuchi patterns was proposed by recognizing the symmetry axis though Kikuchi patterns and using the law of crystal symmetry. The symmetry and crystal structure of three unknown samples were successfully determined by this method. One of the samples was identified to the right crystal system that it naturally belongs, and the other two were fixed on some certain point groups. Through the determination of crystal system and point group, some phase identification results which do not conform to symmetry were excluded. It is indicated that the symmetry analysis of Kikuchi patterns is an effective way to identify the crystal structure and can significantly filter the phase database. Compared with the existing methods, this method greatly reduces the retrieval range of phase identification, and significantly improves the accuracy and reliability of phase identification. It is a promising indexing technique for the new generation of EBSD devices.

收稿日期：2021-02-07; 收到修改稿日期：2021-02-27; 网络出版日期：2021-03-15

基金项目：上海市无机非金属材料分析测试专业技术服务平台(19DZ2290700) Shanghai Technical Platform for Testing on Inorganic Materials (19DZ2290700)

作者简介: 彭 帆(1993-), 女, 博士研究生. E-mail: pengfan@student.sic.ac.cn PENG Fan(1993-), female, PhD candidate. E-mail: pengfan@mail.sic.ac.cn

通信作者：曾 毅, 研究员. E-mail: zengyi@mail.sic.ac.cn ZENG Yi, professor. E-mail: zengyi@mail.sic.ac.cn 
Key words: EBSD; Kikuchi patterns; crystal structure identification; phase identification

电子背散射衍射(EBSD)作为一种相对较新的 显微结构分析手段, 可以在较大范围内提供材料的 取向、织构、相鉴定及含量分布等晶体学统计性信 息 ${ }^{[1-4]}$, 为无机材料制备工艺的改善、微结构调控及 性能优化提供了可靠的科学依据。Fang 等 ${ }^{[5]}$ 通过 EBSD 技术对梯度纳米(GNG)金属铜进行表征, 为 解释其拉伸变形的机理提供了重要的显微结构证 据。EBSD 还是研究涂层内部残余应力分布的最佳 显微结构分析手段, 为研究热障涂层的服役失效行 为提供了重要支撑。EBSD 提供的所有晶体学信息 都依赖对菊池衍射花样的分析 ${ }^{[6]}$, 菊池花样直观反 映材料内部微观结构, 其中, 菊池带对应不同指数 的晶面, 菊池带的交点即菊池极代表晶带轴 ${ }^{[4]}$ 。

相鉴定是 EBSD 的核心功能之一, 商用仪器主 要通过匹配晶面夹角进行相鉴定，可通过成分分析 或人为预先选相来缩小相鉴定的笁选范围。需要选 择的范围越小, 结果就越准确。由于只考虑晶面夹 角这一种信息, 商用 EBSD 存在一定局限性, 有时 会给出多个相鉴定结果, 甚至出现不符合材料对称 性的结果, 容易产生误判。对于这种情况, 单纯依靠 晶面夹角匹配难以排除, 还需对菊池花样进行对称 性分析才能确定。

尽管通过菊池花样的对称性进行相鉴定具有显 著优势, 可有效改善现有方法的局限性, 但目前商 业仪器仍然只采用晶面夹角匹配, 主要原因在于对 称轴的识别非常困难。三维空间的对称轴变成了二 维平面的菊池极, 且伴随难以避免的花样畸变, 增 大了判断难度。虽然一些具有明显几何特征的对称 轴可直接识别, 如六次轴和二次轴, 但不具有明显 特征的对称轴, 或存在与之类似的非对称轴则很难 识别, 需根据对称轴的特点具体分析, 如三次轴和 四次轴。根据晶体对称规律, 交于三次轴的同族晶 面应呈 $120^{\circ}$ 旋转重合, 交于四次轴的同族晶面呈 $90^{\circ}$ 旋转重合。这一规律在菊池花样中表现为: 交于 三次轴的同族菊池带, 其晶面夹角为 $120^{\circ}$, 交于四 次轴的同族菊池带的晶面夹角为 $90^{\circ}$, 以此作为对 称轴的判断依据。准确判断这类对称轴需要判断菊 池带是否属于同一晶面族, 并计算它们之间的晶面 夹角。

菊池带是否属于同一晶面族需计算晶面间距来 判断, Dingley 等 ${ }^{[7]}$ 尝试利用对称性进行相鉴定, 但 因为晶面间距计算误差较大, 只能依靠肉眼在菊池 花样中识别对称轴, 容易误判。通过菊池带宽度计

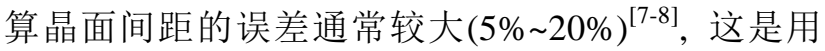
菊池花样进行对称性分析的最大难点。我们前期工 作对晶面间距进行了准确测量, 平均相对误差为 $2.6 \%{ }^{[9]}$, 为准确识别对称轴奠定了基础, 还可区分 非对称轴。对 Si 单晶菊池花样的分析发现了类似三 次轴的非对称轴, 计算晶面间距发现, 菊池带并不 属于同一晶面族, 不满足三次轴的条件, 这是肉眼 识别很容易误判的。晶面夹角可通过三维空间矢量 计算获得 ${ }^{[10]}$, 避免了用菊池带夹角代替晶面夹角所 引入的额外误差。

本研究在准确测量晶面间距的基础上, 结合对 不同点群对称规律的归纳总结, 提出一种简单可行 的利用菊池花样进行对称性分析的方法, 对晶体结 构实现了准确判断。相较于在数据库中检索的传统 相鉴定方法, 对称性分析为相鉴定提供了新思路。 根据对称性排除不可能的晶体结构, 显著缩小了相 鉴定范围, 还可区分常规晶面夹角匹配法无法区别 的结果, 进一步提升相鉴定的可靠性, 是一种有望 用于新一代 EBSD 设备的方法。

\section{1 研究方法}

\section{1 识别对称轴}

对于具有明显几何特征的对称轴(六次轴和二 次轴), 根据其几何特征直接识别。几何特征不明显 的对称轴(三次轴和四次轴)需分析交于该菊池极的 菊池带。如同族菊池带的晶面夹角为 $120^{\circ}$, 判断该 菊池极为三次轴; 如同族菊池带的晶面夹角为 $90^{\circ}$, 判断该菊池极为四次轴。根据计算误差, 将晶面间 距和晶面夹角的容差设定为 $5 \%$ 和 $1^{\circ}$, 即当晶面间 距计算值相差在 $5 \%$ 以内时认为属于同一晶面族, 当晶面夹角计算值相差在 $1^{\circ}$ 以内时认为晶面夹角 相等。

六次轴对应菊池极具有明显几何特征, 在其 周围可见一个或多个由菊池带组成的六边形。四 次轴对应的菊池极, 交于该菊池极的同族菊池带 的晶面夹角为 $90^{\circ}$ 。三次轴对应的菊池极, 交于该 菊池极的同族菊池带的晶面夹角为 $120^{\circ}$ 。二次轴 也具有较为明显的几何特征, 菊池带旋转 $180^{\circ}$ 可 以和自身重合。不同对称轴对应的菊池极如图 1 所示。

以上是对不同对称轴对应菊池极的简单示意, 需要结合菊池花样具体分析。 

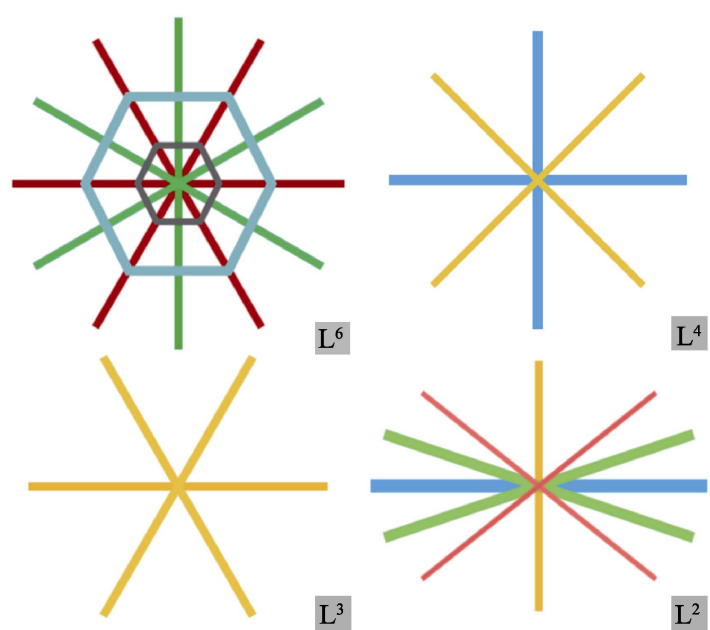

图 1 六次轴、四次轴、三次轴和二次轴对应菊池极的示意图 Fig. 1 Schematic of 6-fold axis, 4-fold axis, 3-fold axis and 2-fold axis

The same color bands represent the Kikuchi bands belonging to the same crystal plane family

\section{2 对称要素组合规律}

对称性是晶体分类的基础，根据对称性晶体可 分为三个晶族(Crystal category)、七个晶系(Crystal system)和 32 个点群(Point group)。不同的点群有各 自的对称要素组合规律(对称要素包括对称轴、对称 面和旋转中心等), 在菊池花样中识别出对称轴以 后, 再根据这些对称轴的组合规律, 对称轴之间的 夹角关系便可以进行对称性鉴定。

以立方晶系中的点群 $m 3 m$ 为例说明。该点群包 含三个四次轴, 四个三次轴, 六个二次轴, 九个对 称面和一个对称中心。这些对称要素之间呈特定的 方式组合, 对称要素之间的组合方式可用极射赤平 投影图表示, $\mathrm{m} 3 \mathrm{~m}$ 点群对称要素的极射赤平投影图 如图 2 所示。

在 $m 3 m$ 点群中, 三次轴和四次轴呈特定的空间 方位组合, 它们之间的夹角只能是 $35.26^{\circ}$ 。不同的

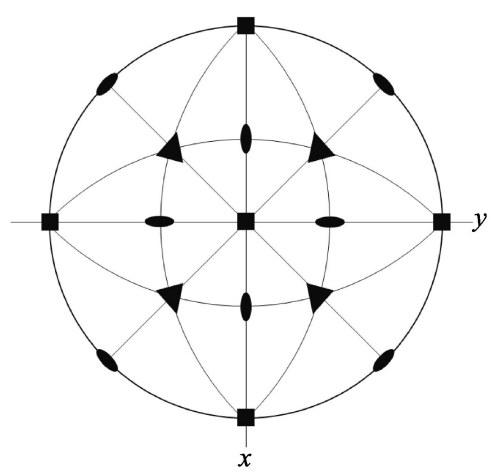

图 2 点群 $\mathrm{m} 3 \mathrm{~m}$ 对称要素极射赤平投影图

Fig. 2 Stereographic projection of symmetry elements of m3m point group

- 4-fold axis; $\Delta$ 3-fold axis; 2-fold axis
晶系, 不同的点群均有各自的对称特点, 且对称要 素之间满足不同的组合规律, 对称轴之间的夹角关 系也不同，这是利用菊池花样进行对称性鉴定的依 据。对称轴只会在晶体的特定方向出现, 例如, 六次 轴只可能是六方晶系中的 $c$ 轴方向, 因此可以将六次 轴标定为[0001]; 再如, 在点群 $\mathrm{m} 3 \mathrm{~m}$ 中, 三次轴和四 次轴只有一种位置关系, 四次轴可标定为 [100], 三次 轴可标定为[111], 而二次轴和四次轴有两种位置关 系, 可标定为[110] $\left(\mathrm{L}^{4} \angle \mathrm{L}^{2}=45^{\circ}\right)$ 或[011] $\left(\mathrm{L}^{4} \angle \mathrm{L}^{2}=90^{\circ}\right)$, 这是利用晶体对称性辅助标定菊池花样的思路。

\section{3 晶体对称性鉴定准则}

在菊池花样中识别出对称轴之后, 结合不同点 群各自的对称特点, 可对花样进行对称性分析和晶 体结构鉴定。受到菊池花样的制约, 不可能所有对 称轴都能在花样中找到, 因此有些花样可以锁定到 部分点群, 有些则只能确定到部分晶系, 而一些没 有出现任何对称轴的花样则不适用于此方法。值得 注意的是, 花样中未发现某种对称轴并不能说明该 材料不具有该对称轴。晶体对称性鉴定流程图如图 3 所示。

下面是对晶体对称性鉴定准则的具体说明。

\subsection{1 出现六次轴}

出现六次轴可判断该晶体属六方晶系。由于六 次轴不可能和三次轴、四次轴同时出现, 只需关注 二次轴。如出现了二次轴则可以进一步将范围缩小 到点群 62 和 6/mmm。由于六次轴和二次轴之间只 可能以 $90^{\circ}$ 相交, 因此无需计算两者的夹角。当然, 计算对称轴间夹角可以验证对称轴判断的准确性, 如计算发现 $\mathrm{L}^{6}$ 和 $\mathrm{L}^{2}$ 之间的夹角不为 $90^{\circ}$, 则说明对 称轴的判断可能有误。

六次轴是晶体中的 $c$ 轴方向, 可将六次轴的菊 池极标定为 [0001], 而二次轴则存在两种类型: 一 种是 $a$ 轴上的二次轴, 另一种则不在 $a$ 轴上。这两

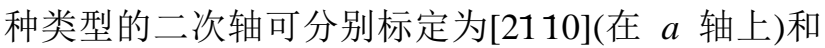
[1010](不在 $a$ 轴上)。此步骤是无法区分这两种二次 轴类型的。

\subsection{2 出现四次轴}

四次轴的出现说明该晶体为四方晶系或立方晶 系。当四次轴和三次轴同时出现时该晶体必为立方 晶系中的点群 43 或 $\mathrm{m} 3 \mathrm{~m}$ 。四次轴和三次轴之间的 夹角只能是 $35.26^{\circ}$, 且彼此呈特定方式组合, 可将 $\mathrm{L}^{4}$ 标定为[100], $\mathrm{L}^{3}$ 标定为[111]。

如未发现 $\mathrm{L}^{3}$ 则接下来关注 $\mathrm{L}^{2}$ 是否存在。 $\mathrm{L}^{3}$ 未 出现不能说明该晶体不存在三次轴, 即不能排除立 方晶系的可能性。如出现 $\mathrm{L}^{2}$ 则需要计算 $\mathrm{L}^{4}$ 和 $\mathrm{L}^{2}$ 之间 


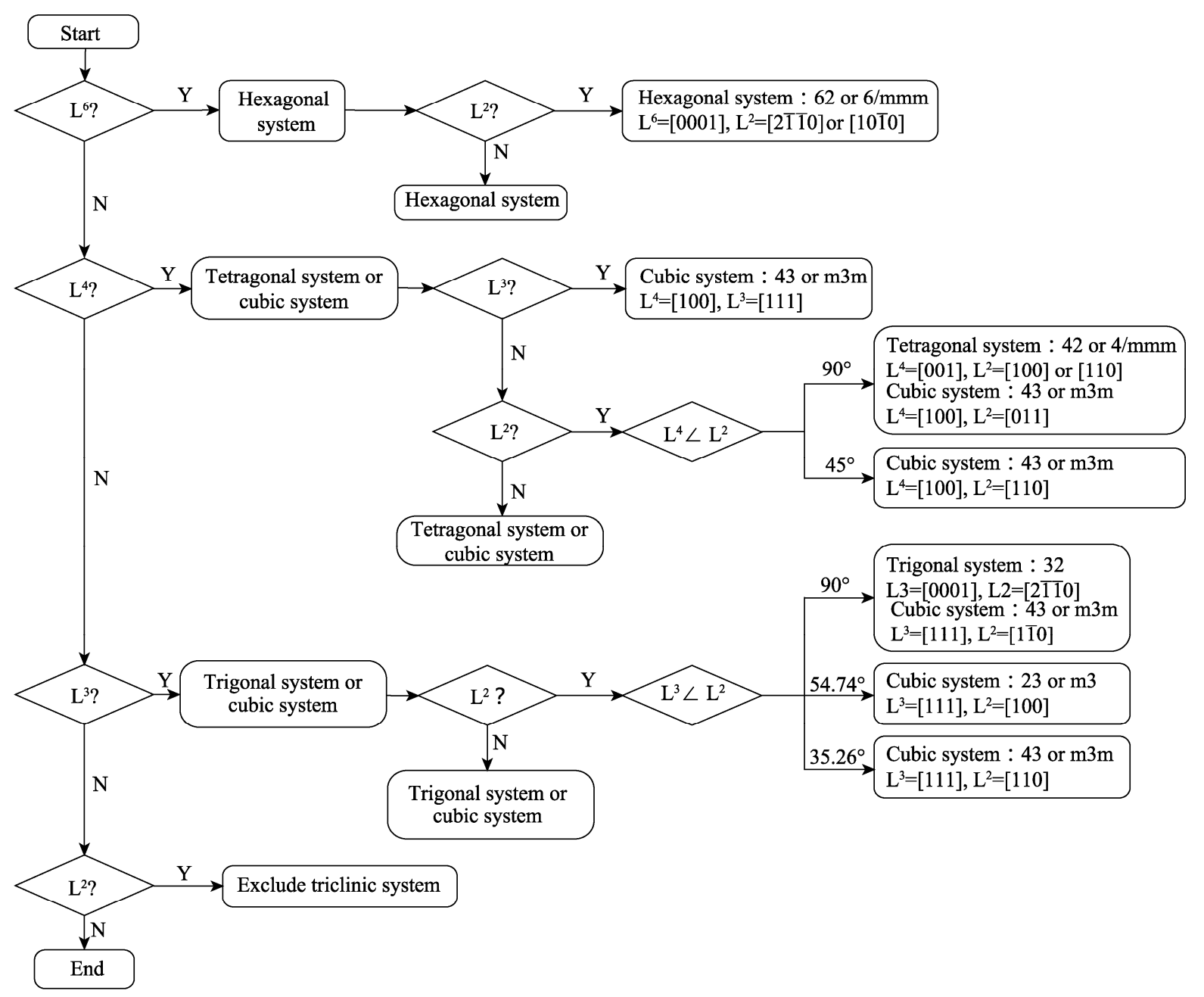

图 3 晶体对称性鉴定流程图

Fig. 3 Flow chart of crystal symmetry identification

的夹角。四方晶系中 $\mathrm{L}^{4}$ 和 $\mathrm{L}^{2}$ 只能以 $90^{\circ}$ 相交, 立方 晶系中 $L^{4}$ 和 $L^{2}$ 的夹角可为 $90^{\circ}$ 或 $45^{\circ}$ 。当夹角为 $45^{\circ}$, 可判断为立方晶系中的点群 43 或 $\mathrm{m} 3 \mathrm{~m}$, 其中 $\mathrm{L}^{4}$ 可 标定为[100], $L^{2}$ 可标定为[110]。当 $\mathrm{L}^{4}$ 和 $\mathrm{L}^{2}$ 的夹角 为 $90^{\circ}$, 则立方和四方都有可能。为四方晶系时, 所 属点群为 42 或 $4 / \mathrm{mmm}, \mathrm{L}^{4}$ 一定是 $c$ 轴方向, 可标定 为[001], 而 $\mathrm{L}^{2}$ 则有两种可能, 分别是[100](在 $a$ 轴 上)和[110](不在 $a$ 轴上); 为立方晶系时, 点群为 43 或 $m 3 m, L^{4}$ 不一定为 $c$ 轴方向, $L^{4}$ 可标定为 $[100], L^{2}$ 可标定为[011]。

\subsection{3 出现三次轴}

出现三次轴可判断该晶体为三方晶系或立方晶 系。如又发现了二次轴, 则可通过 $\mathrm{L}^{3}$ 和 $\mathrm{L}^{2}$ 的夹角进 一步判断。 $\mathrm{L}^{3}$ 和 $\mathrm{L}^{2}$ 的夹角有三种可能性, 分别是 $90^{\circ} 、 54.74^{\circ}$ 和 $35.26^{\circ}$ 。

当夹角为 $90^{\circ}$ 时, 三方和立方均有可能。该晶体 可为三方晶系的点群 32, 其中 $\mathrm{L}^{3}$ 为 $c$ 轴, 可标定为

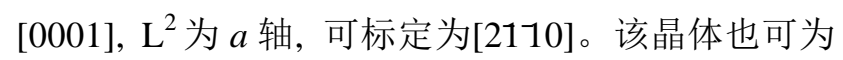
立方晶系中的点群 43 或 $\mathrm{m} 3 \mathrm{~m}$, 其中 $\mathrm{L}^{3}$ 可标定为

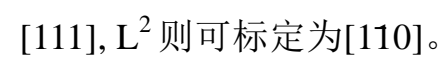

夹角为 $54.74^{\circ}$ 时只可为立方晶系, 且属于点群 23 或 $m 3$, 其中 $L^{3}$ 可标定为[111], $\mathrm{L}^{2}$ 可标定为[100]。 夹角为 $35.26^{\circ}$ 时, 同样只能是立方晶系, 属于点 群 43 或 $\mathrm{m} 3 \mathrm{~m}, \mathrm{~L}^{3}$ 可标定为[111], $\mathrm{L}^{2}$ 则标定为[110]。

\subsection{4 出现二次轴}

出现二次轴可排除三斜晶系。

\section{2 实验结果}

选取三个未知样品(样品 $\mathrm{a}$ 、样品 $\mathrm{b}$ 、样品 $\mathrm{c}$ ) 获 取多幅菊池花样, 已知每个样品只含有一种相。通 过装配在扫描电镜上的牛津 EBSD 系统采集菊池花 样, 加速电压为 $20 \mathrm{kV}$ 。对上述三个未知样品进行对 称性分析和晶体结构鉴定。根据对称轴对应菊池极 的几何特征, 必要时结合菊池带夹角关系和晶面族 关系, 对所获取的菊池花样中的对称轴进行了识 别。对称轴之间的夹角可以通过菊池极坐标进行矢 量计算获得。对称轴的识别结果如图 4 所示。 

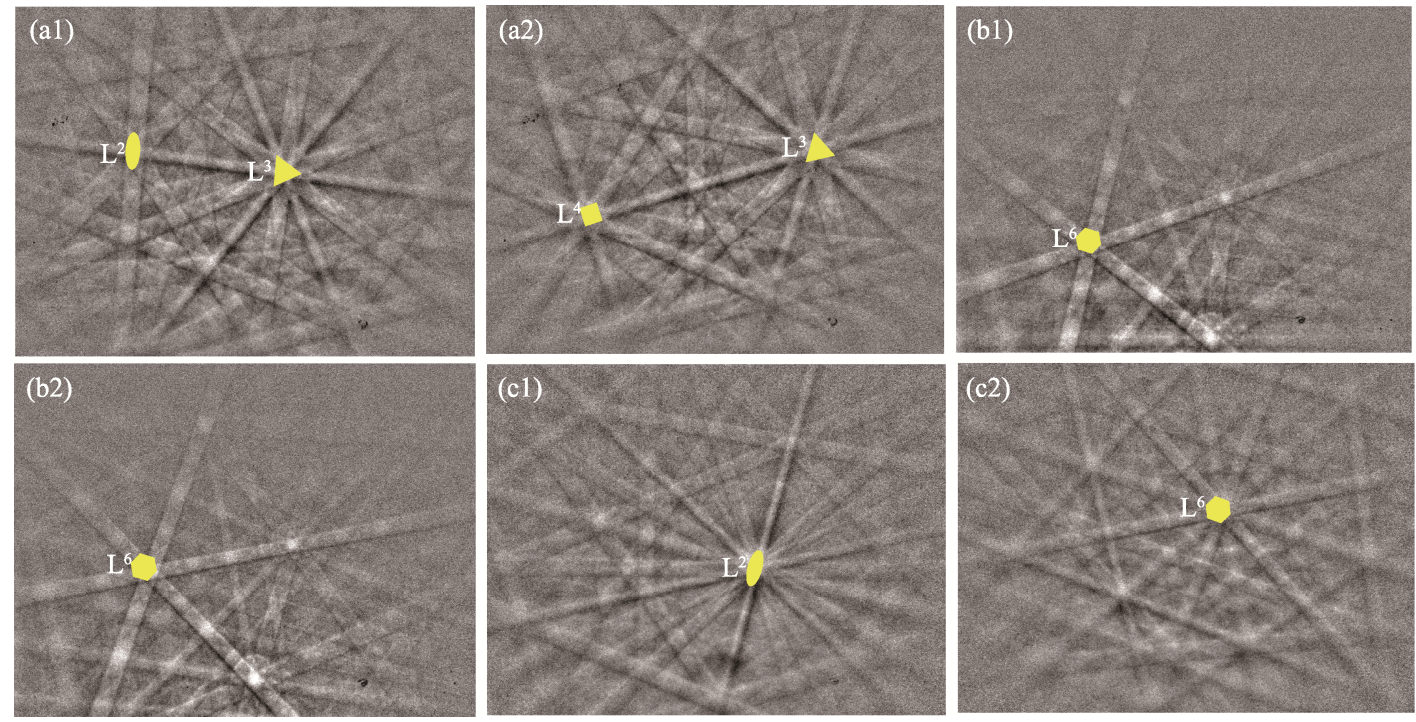

图 4 对称轴识别结果

Fig. 4 Recognition results of the symmetry axis (a1, a2) Sample a; (b1, b2) Sample b; (c1, c2) Sample c

对这些花样分别进行分析。由于花样 a1 和 a2 属于同一相, 两者的晶体结构应一致。a1 中同时出 现了三次轴和二次轴, 根据图 3 所示的晶体对称性 鉴定流程图, 可知 a1 属三方或立方晶系, 进一步计 算三次轴和二次轴之间的夹角可将其判断为立方晶 系中的点群 43 或 m3m。a2 中同时出现了四次轴和 三次轴, 无需计算对称轴间夹角, 可判断为立方晶 系中的点群 43 或 $\mathrm{m} 3 \mathrm{~m}$, 该结果与 a1 的判断结果相 吻合。花样 b1 和 b2 中均出现了六次轴, 由此可判 断样品 $\mathrm{b}$ 为六方晶系。由于没有发现其他对称轴, 无 法进一步缩小范围。花样 c1 中出现了一个二次轴, 据此可以排除三斜晶系。花样 c2 则出现了六次轴, 可判断为六方晶系。虽然二次轴和六次轴并未在同 一花样中同时出现, 但花样 $\mathrm{c} 1$ 和 $\mathrm{c} 2$ 属于同一相, 说 明样品 c 同时含有六次轴和二次轴, 可进一步将范 围缩小到六方晶系中的点群 62 或 $6 / \mathrm{mmm}$ 。

为了进一步对结果进行验证, 本课题组通过商 用 EBSD 获取了三个样品的相鉴定结果。样品 $\mathrm{a}$ 为 点群 $\mathrm{m} 3 \mathrm{~m}$ 的 $\mathrm{Fe}$, 通过对称性分析锁定了点群 43 和 $\mathrm{m} 3 \mathrm{~m}$, 结果正确。样品 $\mathrm{b}$ 为点群 $6 / \mathrm{mmm}$ 的 $\mathrm{SiC}$, 可判 断其为六方晶系, 结果正确。仪器对样品 $\mathrm{c}$ 给出了多 个结果: Ti-Hex(点群为 $6 / \mathrm{mmm}$, 六方晶系) 和 $\mathrm{TiBr}_{4}$ (点 群为 $2 / \mathrm{m}$, 单斜晶系)。而根据对菊池花样的对称性 分析可知, 样品 $\mathrm{c}$ 的菊池花样中出现了六次轴(花样 c2)。根据对称定律, 六次轴的出现只可能是六方晶 系, 因此可以排除仪器给出的 $\mathrm{TiBr}_{4}$ 这一结果。

\section{3 讨论}

在利用菊池花样进行对称性分析的过程中并未
使用对称面等对称要素, 只用到了对称轴。根据对 不同点群对称特点的分析发现, 只使用对称轴已经 可以达到较好的效果, 如果引入对称面的判断, 不 仅会增加判断的难度和工作量, 而且不会对结果产 生较大帮助。

该方法成功应用的一个前提是花样中需要出现 对称轴, 即使只出现一个对称轴也可以帮助缩小篮 选范围。一般来说, 一幅花样中不会出现所有的对 称轴, 这种情况下就可以用属于同一相的多幅花样 进行综合分析判断。例如对样品 c, 虽然二次轴和六 次轴并未同时出现在一幅花样中, 但由于它们属于 同一相, 说明样品 c 同时含有六次轴和二次轴, 这 样便将范围从六方晶系进一步缩小到点群 62 和 6/mmm。当使用属于同一相的多幅花样进行综合分 析时, 还应检验不同花样得到的结果是否相互吻 合。例如花样 a1 和 a2 虽然出现了不同的对称轴组 合, 但最后得到的结果是一致的。

商用仪器在相鉴定过程中并未考虑晶体对称性, 依靠晶面夹角匹配从数据库中检索, 而且识别的条 带数和匹配的晶面夹角数也有限, 点群为 $6 / \mathrm{mmm}$ 的 Ti-Hex 和点群为 $2 / \mathrm{m}$ 的 $\mathrm{TiBr}_{4}$ 两种相均能满足样品 $\mathrm{C}$ 的夹角关系, 导致出现了多个结果。它们虽然用常规 的晶面夹角匹配法无法排除, 但明显不符合样品 c菊 池花样呈现的对称特征，应用对称性予以排除。

晶面夹角匹配法作为目前商用 EBSD 相鉴定的 主要方法, 适用于绝大部分材料, 具有原理简单, 快速高效的优点。但该方法往往会给出多个相鉴定 结果, 甚至出现不符合材料对称性的结果, 影响了 相鉴定的准确性。对称性分析可作为现有晶面夹角 
匹配法的重要补充, 该方法适用于出现对称轴的菊 池花样, 可直接对花样进行对称性分析判断晶体结 构, 显著缩小了相鉴定的笁选范围。该方法还可区 分一些常规晶面夹角匹配法无法区别的结果, 从而 提高相鉴定的准确性和可靠性。

\section{4 结论}

本研究提出了一种利用菊池花样进行晶体结构 鉴定的方法, 根据不同点群对称要素的组合规律建 立了晶体对称性鉴定的流程图。运用上述方法依靠 对菊池花样的分析而无需借助庞大的数据库, 成功 对三个未知样品进行了对称性分析和晶体结构判断, 避免了繁重的检索工作。其中样品 $\mathrm{a}$ 判断为立方晶 系中的点群 43 或 $\mathrm{m} 3 \mathrm{~m}$, 样品 b 判断属于六方晶系, 样品 c 判断为六方晶系中的点群 62 或 6/mmm, 经 过验证结果均正确, 并且排除了商用 EBSD 给出的 相鉴定结果中不满足晶体对称性的结果。说明利用 菊池花样进行对称性分析是判断晶体结构的有效方 法, 可以显著缩小相鉴定的篮选范围, 排除不符合 材料对称特点的结果。该方法可作为现有晶面夹角 匹配法的重要补充, 在进行晶面夹角匹配之前先对 菊池花样进行对称性分析判断晶体结构, 预先确定部 分晶系或者点群, 以缩小笁选范围, 有助于获得更准 确的相鉴定结果。对菊池花样的深入分析有望进一步
挖掘 EBSD 技术在无机材料领域的应用潜力。

\section{参考文献:}

[1] MICHAEL J R. Phase identification using electron backscatter diffraction in the scanning electron microscope, in: SCHWARTZA J, KUMAR M, ADAMS B L (Eds.) Electron Backscatter Diffraction in Materials Science. New York: KluwerAcademic/Plenum Publications, 2000: 75-89.

[2] KUNZE K, WRIGHT S I, ADAMS B L, et al. Advances in automatic EBSP single orientation measurements. Textures and Microstructures, 1993, 20(1-4): 41-54 .

[3] WILKINSON A J, BRITTON T B. Strains, planes, and EBSD in materials science. Materials Today, 2012, 15(9): 366-376.

[4] SCHWARZERR A. Automated crystal lattice orientation mapping using a computer-controlled SEM. Micron., 1997, 28(3): 249-265.

[5] FANG T H, LI W L, TAO N R, et al. Revealing extraordinary intrinsic tensile plasticity in gradient nano-grained copper. Science. 2011, 331(6024): 1587-1590.

[6] WRIGHT S I, ADAMS B L. Automatic-analysis of electron backscatter diffraction patterns. Metall. Trans. A, 1992, 23(3): 759-767.

[7] DINGLEY D J, WRIGHT S I. Determination of crystal phase from an electron backscatter diffraction pattern. J. Appl. Crystallogr., 2009, 42: 234-241.

[8] HAN M, ZHAO G M, ZHU Y. Accurate determination of low-symmetry Bravais unit cells by EBSD. Ultramicroscopy, 2018, 195: 136-146.

[9] PENG F, ZHANG Y, ZHANG J, et al. A study on the indexing method of the electron backscatter diffraction pattern assisted by the Kikuchi bandwidth. J. Microsc-Oxford, 2020, 277(1): 3-11.

[10] ZHANG Y S, FANG S Q, LIN C C, et al. A new method for locating Kikuchi bands in electron backscatter diffraction patterns. Microsc. Res. Techniq., 2019, 82(12): 2035-2041. 